\title{
TRANSIENT HEAT TRANSFER THROUGH WALLS AND THERMAL BRIDGES. NUMERICAL MODELLING: METHODOLOGY AND VALIDATION
}

\author{
Fabrizio Ascione \\ Università degli Studi del Sannio - DING \\ Piazza Roma 21 \\ 82100 Benevento, ITALY \\ Filippo de' Rossi \\ Università degli Studi del Sannio - DING \\ Corso Garibaldi 107 \\ 82100 Benevento, ITALY
}

\author{
Nicola Bianco \\ Università degli Studi di Napoli Federico II - DETEC \\ Piazzale Tecchio 80 \\ 80125 Napoli, ITALY \\ Giuseppe Peter Vanoli \\ Università degli Studi del Sannio - DING \\ Piazza Roma 21 \\ 82100 Benevento, ITALY
}

\begin{abstract}
The current advanced numerical codes for the energy audits carry out 0-dimensional simulation (i.e., one computational node representing the thermal zone), underestimating the effects of thermal bridges on the seasonal heating demand of buildings. The paper suggests a numerical resolution model, implemented in Matlab, aimed to be transferred in numerical engines for the hourly energy simulation. The proposed methodology solves common thermal bridges in buildings, evaluating their effects on the energy demand. Typical thermal bridges have been studied and implemented, analyzing the reliability of the methodology, in terms of accuracy, computational time, required sources, comparing the solutions with those derived by computational fluid dynamic codes. The method reveals very satisfactory results, both as regards the computational time and CPU sources required, as well as with reference to the reliability. Moreover, the solution stability is commonly very high, regardless the chosen computational time-step.
\end{abstract}

\section{INTRODUCTION}

Thermal bridges are critical parts of buildings, being envelope areas characterized by heat losses usually higher than those interesting the common walls. In particular, a lost of one-dimensionality of the heat transmission happens, as shown in Figure 1. This phenomenon, induced by material discontinuity and shape, induces uncontrolled thermal losses and hygiene problems, with possible formation of vapour condensations and mould. Numerically, it was estimated that thermal bridges can increase till $20 \%$ thermal loads and energy needs of buildings, according to various international references as, for instance, the French CSTB (2000).

Kosny and Christian (1995) demonstrated that the thermal resistance of a concrete wall reinforced with steel profiles may diminish also around the $48 \%$, because of the iron thermal bridge effects. Furthermore, Kosny and Kossecka (2002) showed that the calculation of a thermal resistance of a wall, neglecting the thermal bridges and adopting the one-dimensional approach, can induce underestimation around the $44 \%$. The same authors developed the theory of the multi-layer equivalent structure, which allows the investigation of equivalent building envelope, characterized by the same thermal behaviour of the original one.

Several studies have been carried out to optimize building technologies and materials, with reference to glazed facades and transparencies (Ben-Nakh 2002) and opaque structures (Moralesa et al. 2011), in order to minimize the thermal bridges. Despite of this, still today, many softwares for the building energy performance simulation - as, for instance, DOE-2 (Hirsch et al. 2005) or EnergyPlus (U.S. Department of Energy 2010) - solve energy balances through the building envelope recurring to a one-dimensional calculation, without corrections related to thermal bridges. On the other hand, investigations and optimizations require proper calculation methodologies. The scientific literature suggests various and 
different approaches regarding the analysis of thermal bridges, according to both statistical and numerical approaches.

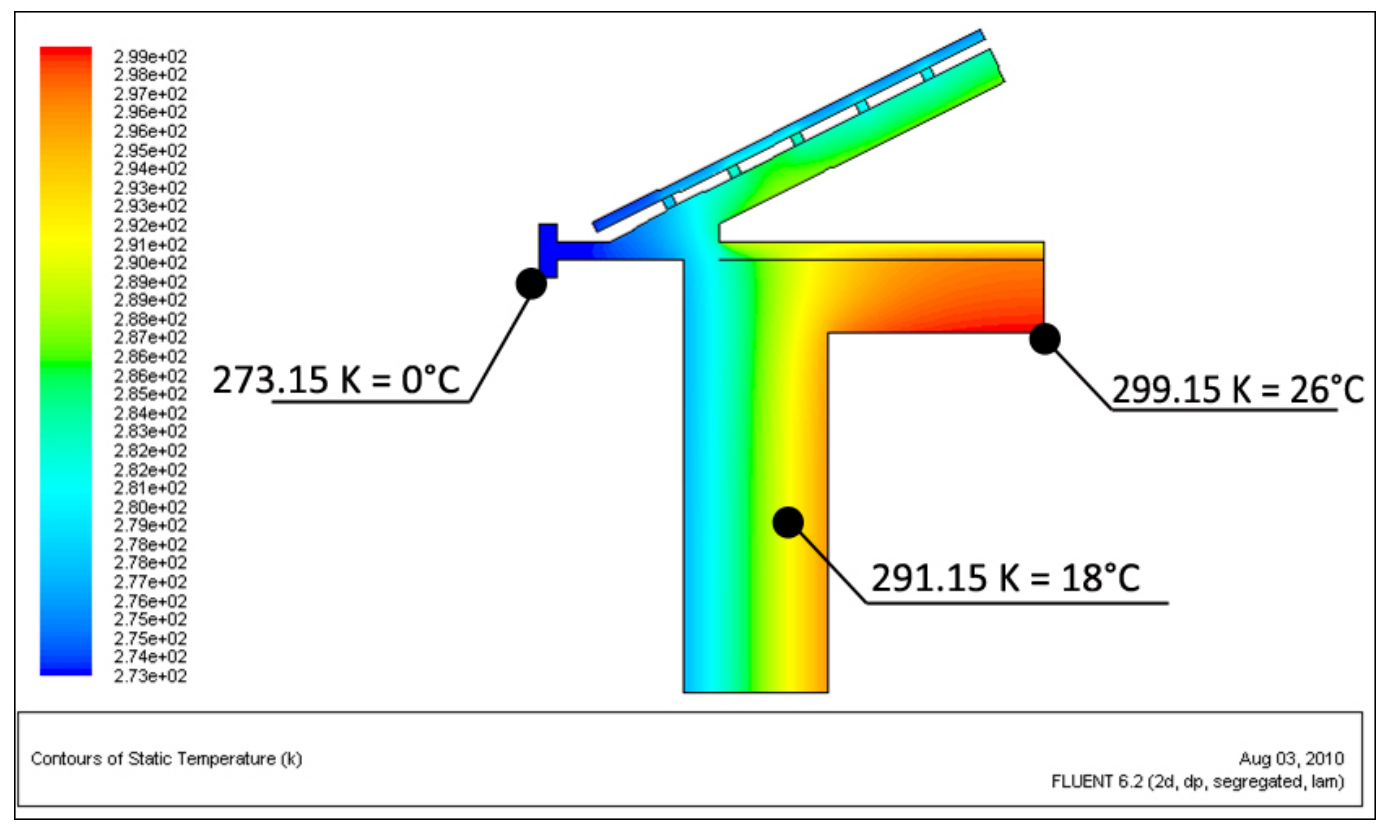

Figure 1: Thermal fields through thermal bridges of the building envelope

Already at the begin of 70s, Mitalas and Stephenson (1971) purpose a method for deriving the transfer functions for one-dimensional flow in multilayer structures, recurring to the Laplace Transform. The LP method was then implemented into the software Blast (Building Loads Analysis and System), starting by the methodologies developed by Hittle (1981) and Hittle and Bishop (1983). In the same years, Seem et al. (1981), Ceylan and Meyers (1980) and Ouyang and Haghigat (1991) implemented the use of CTF Conduction Transfer Functions - for the solution of the transient heat transfer phenomena.

Ceylan and Myers observe the reliability of the numerical method compared to the analytical solution, finding a difference lower than the $1 \%$ as regards the heat transfer evaluation through a flat slab. Finally, Renon (2002) upgraded the methods developed by Seem et al. (1981) and Seem (1987), by modelling thermal bridges and explaining how transfer this technique into the solver engine of EnergyPlus. Deque et al. $(2000,2001)$ presented a numerical methodology developed in order to analyze the heat flow transmitted through a thermal bridge, characterized by state-space representation.

Ben Larbi (2005) developed statistical models useful for the evaluation of the equivalent thermal transmittance of two-dimensional thermal bridges, proposing a library with common geometries.

The approach developed by Seem is the theoretical foundation of the method reported in this paper too. The presented model, as in the following described, allows a very satisfactory reliability and, at the same time, is very useful for reducing the computational power required and the simulation time.

A first investigation concerns a vertical wall analysed adopting one-dimensional approach. Then, the calculation has been extended to a "shape" thermal bridge, induced by the corner joint between orthogonal walls. The analyses are carried out taking into account variability of ambient temperature and solar radiation.

\section{NUMERICAL MODEL}

The thermal field is expressed by the solution of the energy conduction equation, as reported by White (1998) and here proposed in (1)

$$
\frac{\partial}{\partial x}\left(k \frac{\partial T}{\partial x}\right)+\frac{\partial}{\partial y}\left(k \frac{\partial T}{\partial y}\right)+\frac{\partial}{\partial z}\left(k \frac{\partial T}{\partial z}\right)+\dot{q}=\rho c_{v} \frac{\partial T}{\partial \tau} .
$$

Assuming $\mathrm{k}, \rho$ and $\mathrm{c}_{\mathrm{v}}$ as constant, with reference to the temperature and by considering no internal heat source (i.e., $\dot{q}=0$ ), the (2) is achieved, where $\alpha$ is the material thermal diffusivity, usually defined as $\alpha=$ $\lambda /\left(\rho c_{v}\right)$ 


$$
\alpha\left(\frac{\partial^{2} T}{\partial x^{2}}+\frac{\partial^{2} T}{\partial y^{2}}+\frac{\partial^{2} T}{\partial z^{2}}\right)=\frac{\partial T}{\partial \tau} .
$$

By considering the building structures investigated in this study - i.e. a building component largely extended along the $z$ direction, characterized by uniform thermal conditions as regards the surface temperature and the incident solar radiation - the gradient component along the $\mathrm{z}$-axis becomes negligible. Thus, the term $\left(\delta^{2} T / \delta^{2} z\right)$ can be neglected. Therefore, (2) can be simplified into (3)

$$
\alpha\left(\frac{\partial^{2} T}{\partial x^{2}}+\frac{\partial^{2} T}{\partial y^{2}}\right)=\frac{\partial T}{\partial \tau} .
$$

The boundary conditions, at the begin of the simulation, require tailored definitions. In the following of this paper, a constant starting temperature, called $\mathrm{T}_{0}$, has been considered.

The achievement of the solution, by using analytical methods, is very complex. For this reason, numerical methods based on FEMs (i.e., finite differences methods) are commonly used. The first step requires the domain discretization, in order to pass from a system of differential equations to a group of algebraic correlations. Scientific literature offers various techniques for the solution of the mathematical system. These adopted methodologies affect the required quality of the computational grid and, thus, the computational time and power. The computational time and the used computational power are, commonly, the greatest problems, that do not allow such simulations referred to the whole heating/cooling seasons. By considering the above-reported difficulties, Seem (1987) suggested an alternative approach, by means of a model based on the conduction transfer functions.

The first step is the discretization of the geometrical domain into a defined number of nodes. Then, energy balances are performed with reference to each node of the computational grid. The balance equations define a system of differential equations, ordinary and of the first order. The state-space representation, with reference to a system characterized by " $n$ " state variables, " $p$ " inputs and " $m$ " outputs, is proposed in the (4) and (5)

$$
\left\{\begin{array}{l}
\frac{d x}{d \tau}=A x+B u \\
y=C x+D u
\end{array}\right.
$$

where

- A is a matrix of constant terms, with dimensions $(n \times n)$;

- B is a matrix of constant terms, with dimensions $(n \times p)$;

- $\mathrm{C}$ is a matrix of constant terms, with dimensions $(m \times n)$;

- $\mathrm{D}$ is a matrix of constant terms, with dimensions $(m \times p)$;

- $\mathrm{y}$ is the vector of the $m$ outputs;

- $\mathrm{u}$ is the vector of the $p$ inputs;

- $\quad \mathrm{x}$ is the vector of the $n$ state-variables;

- $\tau$ is the time.

After the domain discretization, the elements of the matrices A, B, C, and D are independent of the considered time-step and of the system inputs. About the solver procedures, Brogan (1991) provides the solution for a system of first order differential equations, with constant coefficients (6)

$$
x_{t+\delta}=e^{A \delta} x_{t}+\int_{t}^{t+\delta} e^{A(t+\delta-\tau)} B u(\tau) d \tau .
$$

where

- $\delta$ is the time step [h];

- $u(\tau)$ is the input between the instant $t$ and $t+\delta$.

The term $u(t)$ is determined by recurring to (7)

$$
u(\tau)=u_{\tau}+\frac{(\tau-t)}{\delta}\left(u_{t+\delta}-u_{t}\right)
$$


Moreover, (8) and (9) are obtained by substituting the (7) into (6)

$$
\begin{gathered}
x_{t+\delta}=e^{A \delta} x t+\int_{t}^{t+\delta} e^{A(t+\delta-\tau)} B\left[u_{t}+\frac{\tau-t}{\delta}\left(u_{t+\delta}-u_{t}\right)\right] d \tau . \\
x_{t+\delta}=e^{A \delta} x_{t}+\left[\int_{0}^{\delta} e^{A(\delta-\alpha)} d \alpha\right] B u_{t}+\left[\int_{0}^{\delta} \alpha e^{A(\delta-\alpha)} d \alpha\right]\left[\frac{B}{\delta}\left(u_{t+\delta}-u_{t}\right)\right] .
\end{gathered}
$$

The solution of the first integral of (9) is reported in (10). The solution of the second integral is defined in (11)

$$
\begin{gathered}
\int_{0}^{\delta} e^{A(\delta-\alpha)} d \alpha=A^{-1}\left(e^{A \delta}-I\right) . \\
\int_{0}^{\delta} \alpha e^{A(\delta-\alpha)} d \alpha=A^{-1} A^{-1}\left(e^{A \delta}-I\right)-A^{-1} \delta .
\end{gathered}
$$

Once defined the terms reported into (12), (13) and (14), then the vectors of the variables at the instant $t+\delta$ may be obtained, according to (15)

$$
\begin{gathered}
\Phi=e^{A \delta} . \\
\Gamma_{1}=A-1(e A \delta-I) B . \\
\Gamma_{2}=\left[A^{-1} A^{-1}\left(e^{A \delta}-I\right)-A^{-1} \delta\right] \frac{B}{\delta}=A^{-1}\left(\frac{\Gamma_{1}}{\delta}-B\right) . \\
x_{t+\delta}=\Phi x_{t}+\left(\Gamma_{1}-\Gamma_{2}\right) u_{t}+\Gamma_{2} u_{t+\delta} .
\end{gathered}
$$

The last term of (15) requires the knowledge of the input that characterizes the next instant (i.e., $t+\delta$ ). Seem (1987), in order to by-pass this numerical problem, proposed the (16)

$$
y_{t}=\sum_{j=0}^{n} S_{j} u_{t-\delta j}-\sum_{j=1}^{n} e_{j} y_{t-\delta j}
$$

where $\mathrm{S}$ is a matrix of dimensions $(m x p)$, and its elements are the terms of the transfer functions, with

$$
\left\{\begin{array}{l}
S_{0}=C R_{0} \Gamma_{2}+D \\
S_{j}=C\left[u_{j-1}\left(\Gamma_{1}-\Gamma_{2}\right)+R_{j} \Gamma_{2}\right]+e_{j} D \quad 1 \leq j \leq n-1 \\
S_{n}=C R_{n-1}\left(\Gamma_{1}-\Gamma_{2}\right)+e_{n} D .
\end{array}\right.
$$

Also $e_{j}$, as it appears in (17), is a term of the transfer function. The calculation of the $\mathrm{n}$ square Rmatrices, characterized by the dimension $\left(\begin{array}{lll}n & x & n\end{array}\right)$ with constant coefficients and $\mathrm{n}$ scalars, is carried out according to the Leverrier's algorithm, as reported by Zheng and Wang (2005).

The main advantages offered by (16) are the connections between the output of the examined instant and the various inputs at the same instant, as well as the connection among the inputs of the past instants and their outputs.

By increasing the node numbers (n), the coefficients of the transfer function reduce their entities together to the $\mathrm{j}$ progression. The calculation of matrices of transfer functions, therefore, is necessary and will affect the computation times and the required computational sources. In this regard, the scientific literature suggests various methods for reducing the equation numbers, in order to limit the necessary power and calculation time, without prejudicing the reliability of the outcomes, as underlined by Marshall (1966) and Moore (1981).

In regard to the evaluations of thermal bridges in building, Deque et al. (2000) suggest methods based on the Moore's study, achieving good results, both with reference to the methodology applicability and the reliability of the achievable results, compared to those obtained by applying much more complex methodologies.

Moreover, Gao et al. (2008) studied a reduction model that, coupled to the evaluation of the onedimensional thermal flux through the building envelope, allows the estimation of the building heating load. This model has been, later on, implemented in TRNSYS (Klein et al. 2000). 
The present study tests the predictive reliability of the method described by Ouyang and Haghigat (1991), starting from (18)

$$
\left\{\begin{array}{l}
x_{t+\delta}=\Phi x_{t}+\left(\Gamma_{1}-\Gamma_{2}\right) u_{t}+\Gamma_{2} u_{t+\delta} \\
y_{t+\delta}=C x_{t+\delta}+D u_{t+\delta} .
\end{array}\right.
$$

In particular, the paper suggests an analysis regarding the method reliability, and, at the same time, evaluates its convenience under the point of view of source required. A first example has been carried out in regard to a simple wall geometry, and thus with reference to a vertical building structure analyzed under the hypothesis of one-dimensional heat flux. Then, the methodology is applied to a thermal bridge, determined by the L-shape consisting into the joint of two orthogonal walls. The studies will provide the convective heat flow on the inner side of the opaque structures.

\section{DESCRIPTION OF THE INVESTIGATED BUILDING STRUCTURES}

Two geometries have been considered: a linear wall (Figure 2) - evaluated under one-dimensional heat flux hypothesis - and a wall angle (L shape), representing the typical example of connections at the building corners (Figure 3). A very rigorous study should have taken into account the 3-D effects due to the thermal bridges. The authors verified that this simplification does not affect the method reliability. In fact, being the structure mainly developed in two dimensions, the evaluated gradient component, along the neglected axis, is not numerically significant.

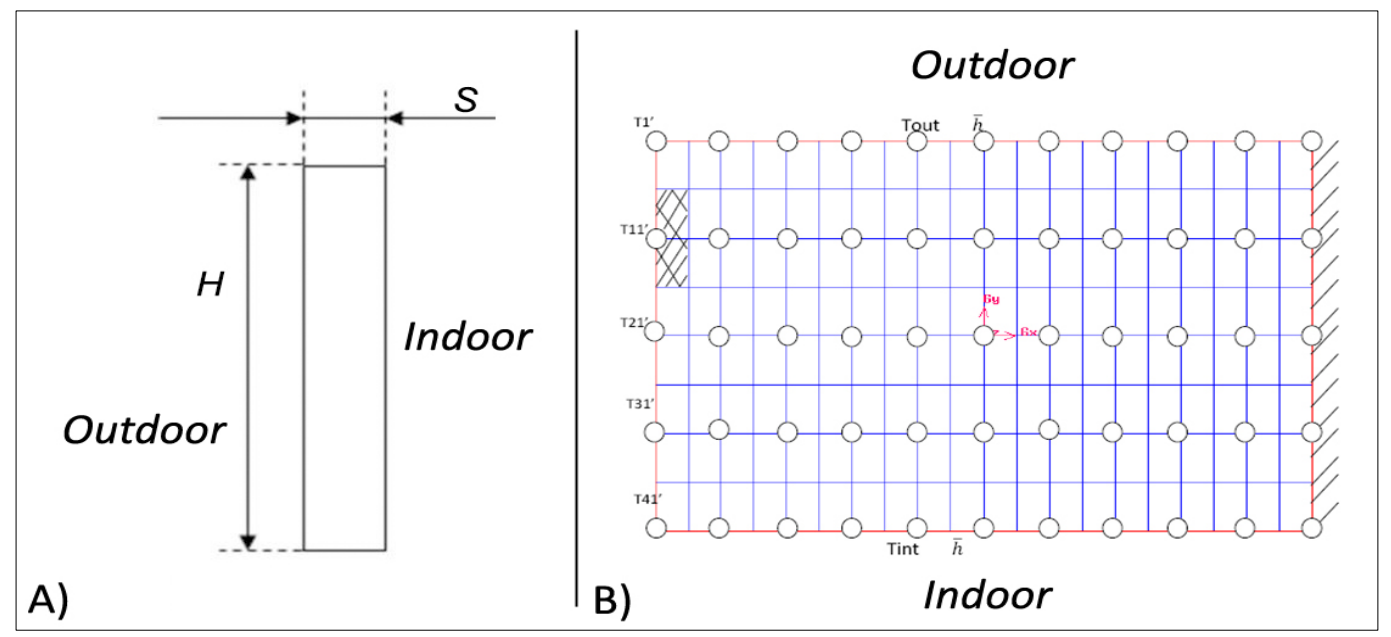

Figure 2: Investigated Geometry 1 (A) and domain discretization for the resolution of thermal field (B)

With reference to the first structure (the linear wall), an envelope component in filled-bricks has been considered, with a total thickness equal to $0.30 \mathrm{~m}$. The wall width and height are quite extended, and therefore the border effects are not significant.

The adopted bricks, very spread as building material, have the thermophysical properties proposed in (19)

$$
k=0.65 \mathrm{~W} /(\mathrm{mK}) \quad \rho=.1750 \mathrm{~kg} / \mathrm{m}^{3} \quad c_{v}=840 \mathrm{~J} /(\mathrm{kgK}) .
$$

Moreover, a second geometry has been studied. A thermal bridge, due to the L-connection between orthogonal walls, has been calculated (the geometry is shown in Figure 3). 


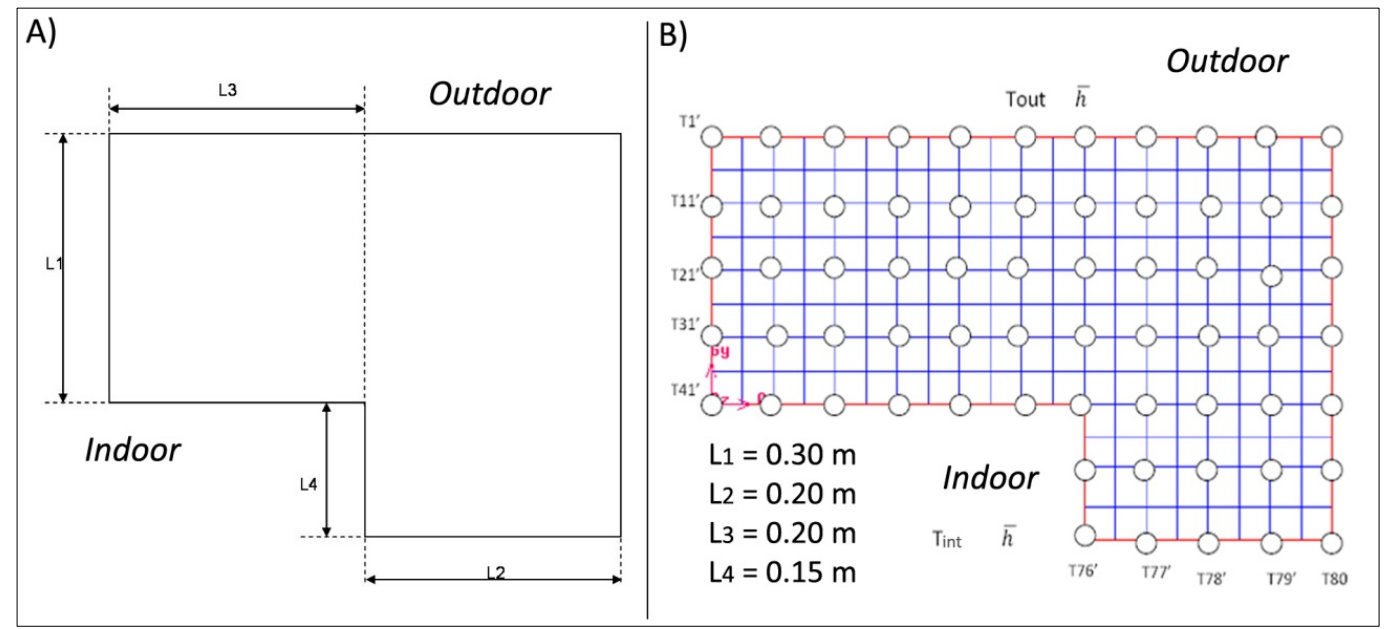

Figure 3: Investigated Geometry 2 (A) and domain discretization for the resolution of thermal field (B)

The convective heat exchange, on both the sides of the walls, has been considered as constant, and equal to $8 \mathrm{~W} / \mathrm{m}^{2} \mathrm{~K}$.

The ambient air temperature has been chosen equal to the $273.15 \mathrm{~K}\left(\rightarrow 0{ }^{\circ} \mathrm{C}\right)$, at the starting condition and, then, in a second stage, it was time-varied, according to a sinusoidal law (Figure 4A).

The inner sides of the wall are characterized by the contact with the indoor air, characterized by a thermal level fixed equal to $300.15 \mathrm{~K}\left(\rightarrow 27^{\circ} \mathrm{C}\right)$, due to the air-conditioning system. In addition, the effects of the solar radiation have been considered, according to the time variation law reported in Figure 4B.

Finally, with reference to the long-wave radiation between the building and the sky, this is not considered, according to the ASHRAE (2009) instructions for vertical components.
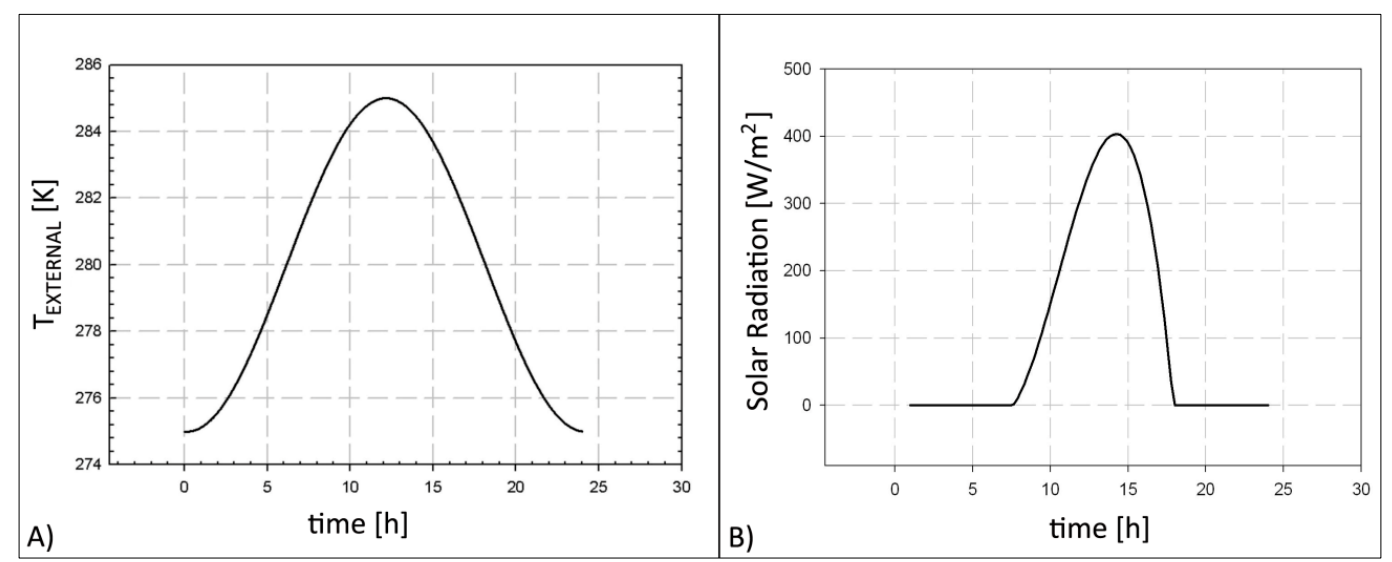

Figure 4: Ambient conditions as regards Temperature (A) and Solar Radiation (B)

\section{DISCUSSION}

\subsection{One-Dimensional Wall}

With reference to the CFD model, the code Ansys Fluent has been used, that implements finite volume algorithms. This is is a powerful instrument for the numerical prediction of thermal, hygrometric and kinetic fields induced by airflows. However, Fluent is very adapt also for the evaluation of the conductive heat transfer through solid structures (Ansys 2005) and it was used for this scope by other authors, also for the thermal bridging evaluation (Martin et al. 2011, Ascione et al. 2012).

The analysis by means of Fluent has been carried out realizing the real geometry with the Gambit application, by varying the meshes (called with the Greek letters " $\alpha$ ", " $\beta ", " \gamma$ " and " $\delta ")$, characterized by 
various numbers of nodes, in order to verify the reliability of the CFD investigations and to assess the impact of the computational grid on the output values.

The grid quality can affect greatly the reliability of a simulation. Grids can be realized in several ways (structured, unstructured, orthogonal or not) and can vary also in the shape of cells (triangular, quadrilateral, tetrahedral and so on). The number of nodes plays a primary role. A tight mesh is required near the domain boundaries and where high gradients of the variable (e.g., the temperature) are expected. Other areas can be discretized with nodes less close. Often, a satisfactory solution begins with a coarse mesh, then adding cells in significant points. Geometrical relations (i.e., aspect ratio, face alignment and cell volume) can preprocess the quality of a mesh. Anyway, the most important verification regards, after the simulations, the independence of the results with change in the computational grid.

As said, the used software is Fluent. Really, several other commercial codes could be used for these comparisons, as, for instance, Comsol Multiphysics or Therm, working under finite elements' modelling. Anyway, when conduction heat transfer phenomena are investigated, both FVM and FEM methods are considered quite reliable.

The main results are reported in Figure 5A, where, the scarce influence of the computational grid can be clearly understood. For the next analyses, the computational grid " $\gamma$ " has been considered (shortly described in Table 1 as regards the cell number).

Table 1: Vertical wall: characteristics of the meshes implemented in Fluent

\begin{aligned} & \hline Name of the Mesh Number of Cells \\ & \hline Mesh " $\beta$ " 6000 \\ & \hline Mesh " $\zeta$ " 15000 \\ & \hline Mesh " $\gamma$ " 24000 \\ & \hline\end{aligned}
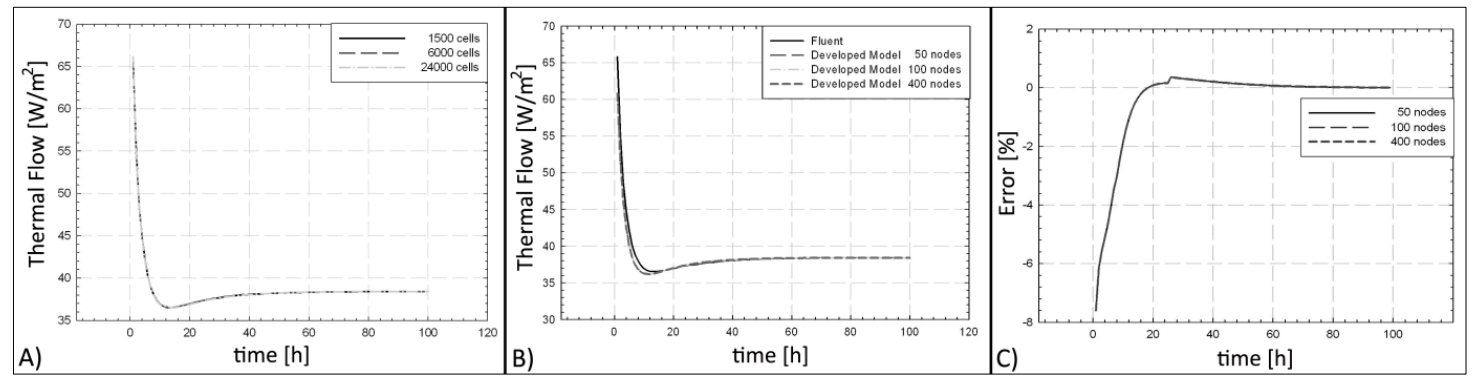

Figure 5: Geometry 1. Time dependent thermal flows (A: Fluent, with change in meshes, B: Fluent vs Model) and percentage error applying the developed model (C). The external temperatures are constant

Figures 5B and 5C show the heat flows calculated recurring to the proposed model implemented in MatLab (2006) and the comparison with the Fluent CFD simulations.

The external temperature has been considered as constant. The figures show also the effects of various discretizations of the domain. It can be noted that, quite always, the curves are very close each others. The absolute detected error is significant only during the initial time-periods, when the proposed model underestimates the heat flows around the $8 \%$. Then, the error resulting adopting the model goes toward a stabilization. After around $50 \mathrm{~h}$, a value of the gap around the $0.1 \%$ is obtained, with respect to the results achieved by means of the Fluent investigations. Starting by the $50^{\text {th }}$ time-step, also the heat flow assumes a quite stable value, around $38.4 \mathrm{~W} / \mathrm{m}^{2}$.

In Figures $6 \mathrm{~A}$ and $6 \mathrm{~B}$, the results, achieved considering the external temperature variation, have been reported. The external temperatures follow the sinusoidal law proposed in (20)

$$
T_{E X T}=280+5 \cos (7.27 e-5 \tau+43200) \text {. }
$$

Again, the proposed model provides satisfactory results. In fact, the percentage difference is more evident during the firsts time-steps, while, when the heat flux goes to the stabilization, the gap is stable around the $-0.7 \%$. Until now, the considered time-interval was established equal to $1 \mathrm{~h}$.

In order to understand the variation of the results with change in time-steps, shorter time-intervals - equal to $0.50 \mathrm{~h}$ and $0.25 \mathrm{~h}$ - have been then considered. During the initial part of the simulations, the gaps, with a 
time step equal to $0.25 \mathrm{~h}$, become lower, being around $-1.8 \%$. On the other hand, the choice of a longer timestep does not affect the method reliability. Indeed, also increasing the time-interval till $2 \mathrm{~h}$, the applied method allows a good correspondence among the results. All the comparisons have been carried out among the MatLab models and the ones implemented in Fluent, obviously considering analogues time-steps.

Table 2 shows the computational times required; the total analysed period covers $50 \mathrm{~h}$, analysed under a time-step equal to $1 \mathrm{~h}$. As expected, an increase of the grid nodes induces the growth of the computational time, being equal to 5.6 seconds for the geometry characterized by 400 nodes. The same analyses, by means of the CFD code Fluent, requires around 318 seconds. For the calculations, the used personal computer has the following peculiarities: PC $1.46 \mathrm{GHz}$, Intel Dual Core, 32 bit, RAM: $2 \mathrm{~Gb}$, OS: Windows Vista.
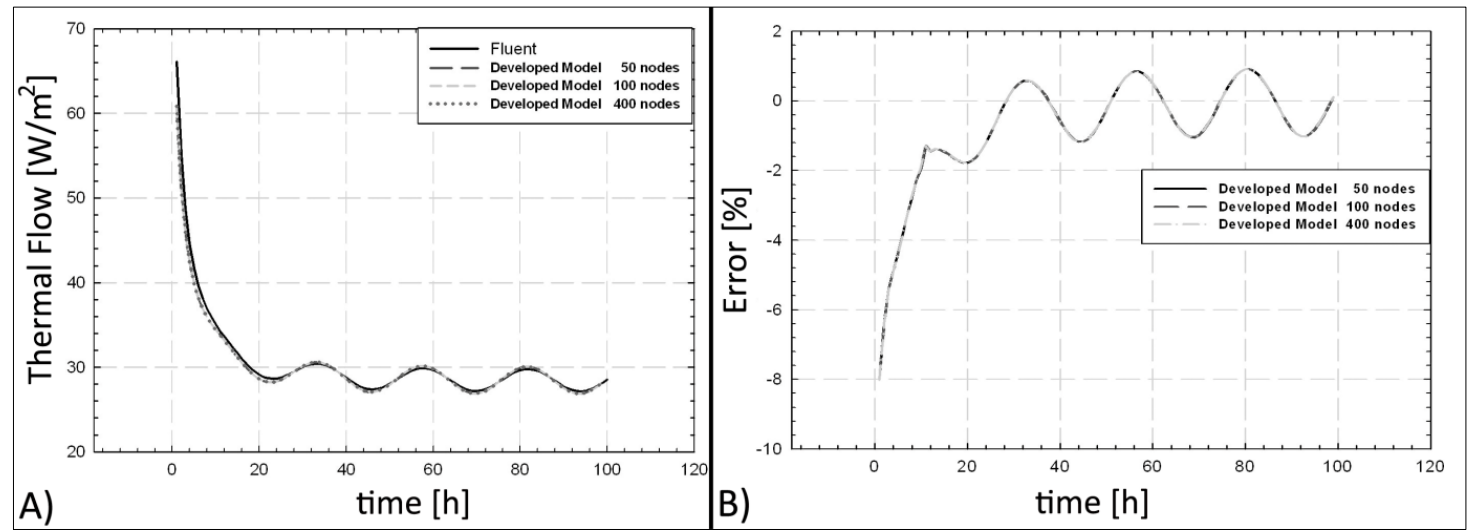

Figure 6: Geometry 1. Time dependent thermal flows, Fluent vs Model (A) and percentage error applying the developed model (B). The external temperatures are variable

Table 2: Vertical Wall: computational times for the simulation converge, with change in node numbers

\begin{aligned} & \hline Characteristics of the Model Computation required time (s) \\ & \hline 50 nodes 2.4 \\ & \hline 100 nodes 3.6 \\ & \hline 400 nodes 5.6 \\ & \hline\end{aligned}

\subsection{Thermal Bridge at the Corner of the Building Envelope}

Since the model - in the first analyses - gave satisfactory outcomes, it was tested also for the study of the heat flow between the inner side of the wall and the indoor air, by considering the L-thermal bridge (Figure 3).

The definition of boundary conditions for the borders of the thermal bridge is necessary. A fixed temperature value on these interfaces has been established, choosing the thermal level of the middle point of the wall, i.e. equal to $286.15 \mathrm{~K}\left(\rightarrow 13{ }^{\circ} \mathrm{C}\right)$. Again with reference to this study, the analysis has been carried out according to different levels. In Table 3, the number of the cells characterizing the computational mesh, then solved with Fluent, is reported. Figure 7A shows the trend of heat flow according to four different discretizations of the domain.

Table 3: L-structured thermal bridge: characteristics of the meshes implemented in Fluent

\begin{tabular}{|c|c|}
\hline Name of the Mesh & Number of Cells \\
\hline Mesh " $\beta$ " & 1500 \\
\hline 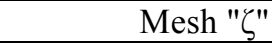 & 4000 \\
\hline Mesh " $\gamma$ " & 6000 \\
\hline Mesh " $\chi$ " & 24000 \\
\hline
\end{tabular}

For the comparisons with the proposed model, implemented in MatLab, it was chosen the " $\gamma$ " mesh. Also with reference to the model here proposed, several numbers of nodes have been considered for the resolution in MatLab. In particular, three cases have been investigated, respectively characterized by 1340, 2100 and 
3000 nodes. In Figures 7B and 7C, the comparisons between the two methodologies are reported, in terms of entity of the thermal flows and percentage gaps, assuming a constant external temperature. With reference to the number of nodes, all the previously-cited solutions have been simulated. It can be noted a constant underestimation of the heat flow by adoption of the proposed numerical method.

The value of underestimation depends, primarily, by discretization and, secondly, by the time-step. When the heat flux assumes a stationary trend, with values around $51.3 \mathrm{~W} / \mathrm{m}^{2}$, the percentage error passes from the $-3 \%$ ( $\rightarrow$ geometry with 1340 nodes $)$ to the $-1 \%$ ( $\rightarrow$ geometry with 3000 nodes).
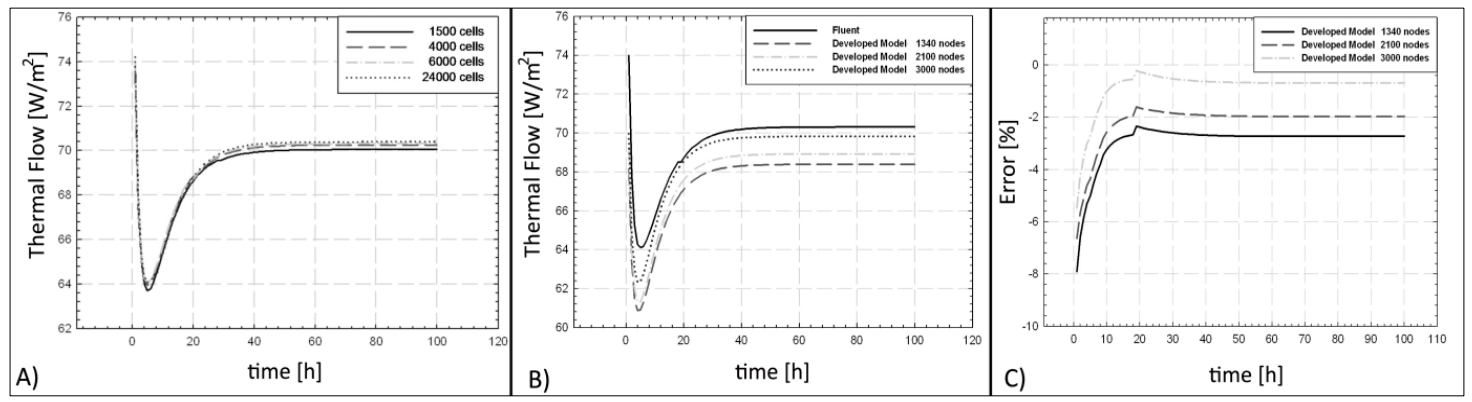

Figure 7: Geometry 2. Time dependent thermal flows (A: Fluent, with change in meshes, B: Fluent vs Model) and percentage error applying the developed model (C). The external temperatures are constant

Furthermore, the ambient temperature variability has been assumed, with the same variation law described in (20). The results are reported in Figures 8A and 8B. Again, the same conclusions of the previous analysis are inferred: an underestimation of the heat flow is verified at the first instants.
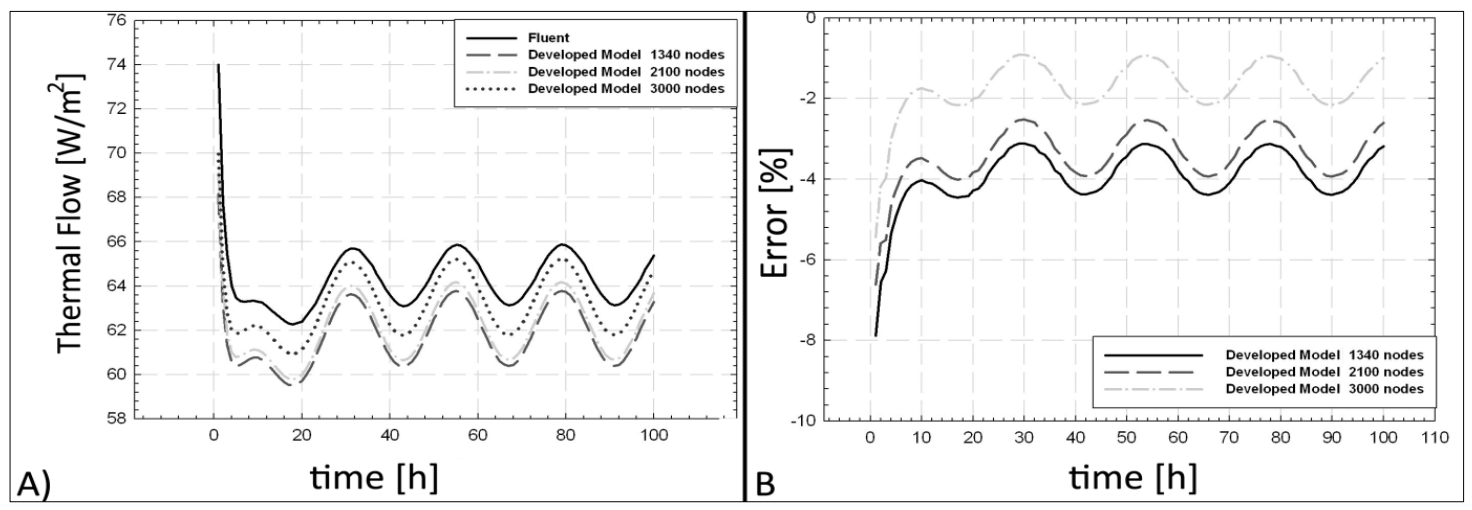

Figure 8: Geometry 2. Time dependent thermal flows (A: Fluent vs Model) and percentage error applying the developed model (B). The external temperatures are variable
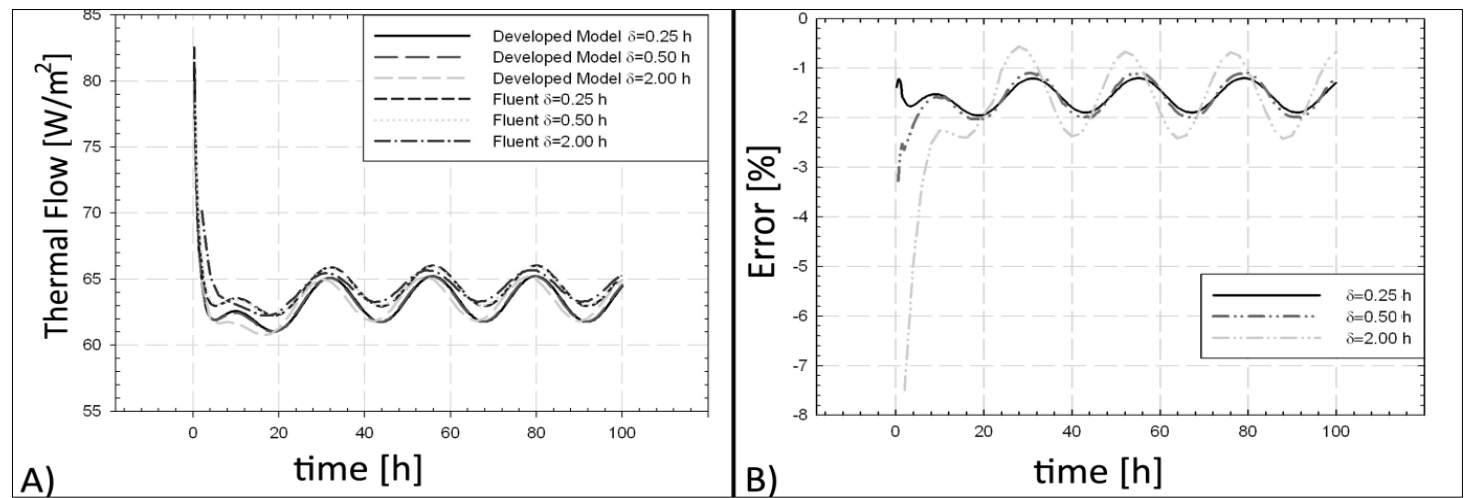

Figure 9: Geometry 2. Time dependent thermal flows (A: Fluent vs Model) and percentage error applying the developed model (B). Various time-step ranges are considered 
Also in this analysis, the next study concerns the consideration of different time-intervals. Comparisons have been carried out by analysing the various outcomes compared to the Fluent investigations, characterized by the same time-step. All the data are reported in Figures 9A and 9B.

For $\delta=0.25 \mathrm{~h}$, the error is very low, being around $-1.5 \%$. A different discussion is necessary for $\delta=2.0 \mathrm{~h}$. In particular, this longer time-step induces a greater error during the first period, but, as expected, no problems of stability occur during the whole analysis.

The incident solar radiation is represented in Figure 4B. With reference to the CFD solution, a dedicated UDF (i.e., User Defined Function), containing all the results, has been realized and implemented in Fluent. In particular, the UDF has been created as a procedure where, once assigned the heat flow for two timeinstants, a linear interpolation is automatically generated every time the heat flux is required for an assigned intermediate value of the time.

The studies have been carried out considering a global time-period equal to 48 hours. In Figures 10A and 10B, it can be inferred that the heat flows - obtained applying the MatLab model and those achieved by the CFD Fluent simulations - differ around $-5 \%$ averagely. The maximum gap is around $-8 \%$. This quite significant underestimation is surely due to the interpolation process.
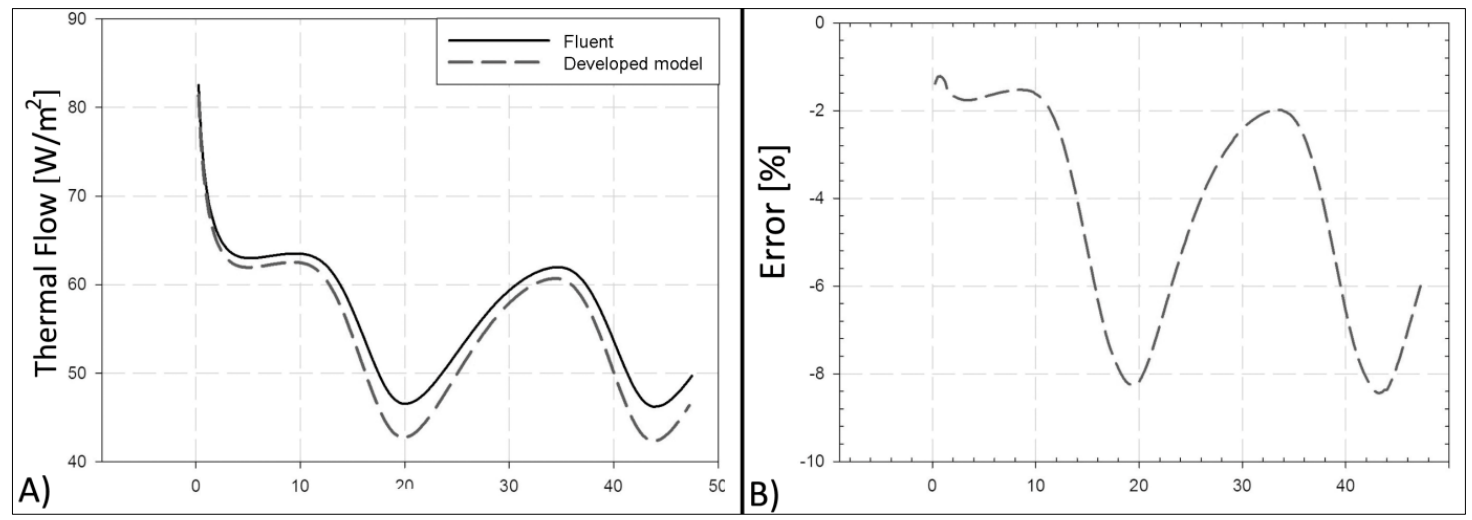

Figure 10: Geometry 2: Time dependent thermal flows (A, Fluent vs Model) and percentage error applying the developed model (B). The solar radiation is varies during the day

The recorded computational times are summarized in Table 4, which shows that, with reference to a number of nodes equal to 1340 , the computational required time is 6.2 seconds, while, if the number of nodes is 3000 , the total request simulation time is 13.2 seconds. Also in this case, the total analysed period covers $50 \mathrm{~h}$, analysed under a time-step equal to $1 \mathrm{~h}$. The same analyses, carried out by means of Fluent, require a computational time at least ten times higher.

By considering that, usually, a building has several dozens of thermal bridges, this outcome shouldn't be underestimated.

Table 4: L-structured bridge: computational times for the simulation converge, with change in node numbers

\begin{aligned} & \hline Characteristics of the Model Computation required time (s) \\ & \hline 1340 nodes 6.2 \\ & \hline 2100 nodes 9.5 \\ & \hline 3000 nodes 13.2 \\ & \hline\end{aligned}

\section{POTENTIAL INTEGRATION IN ENERGY SOFTWARE}

A last section investigates how the proposed methodology could be integrated with hourly energy simulation software, as, for instance, EnergyPlus. This software is a powerful tool when the analysis goal is a full prediction of the whole building-HVAC systems performances, as shown by Pisello et al. (2012) for residential buildings, Assem and Al-Mumin (2010) for offices, Ali Alajm (2012) for Schools, Ascione et al. (2011) for universities. Normally, the building simulators operate, with reference to each specific thermal 
zone, by solving a set of equations that involve the calculation of the convective heat transfer from the zone surfaces, the internal loads, the air mass transfer among zones.

If the air capacitance is neglected, EnergyPlus operates, time-step by time-step, according to (21), in order to provide the zone thermal load and, therefore, the energy required to the HVAC system

$$
-Q_{H V A C}=\sum_{i=1}^{N s j} \dot{Q}_{i}+\sum_{i=1}^{\text {Nsurface }} h_{i} \cdot A_{i} \cdot\left(T_{s i}-T_{z}\right)+\sum_{i=1}^{N z o n e s} m_{i} \cdot c_{p} \cdot\left(T_{z i}-T_{z}\right)+m_{\mathrm{inf}} \cdot c_{p} \cdot\left(T_{\text {ext }}-T_{z}\right) .
$$

The following terms are defined

$$
\sum_{i=1}^{N s j} \dot{Q}_{i}=\text { sum of convective internal loads. }
$$

$$
\begin{gathered}
\sum_{i=1}^{\text {Nsurface }} h_{i} \cdot A_{i} \cdot\left(T_{s i}-T_{z}\right)=\text { convective heat exchange from the zone surfaces. } \\
\sum_{i=1}^{N z o n e s} m_{i} \cdot c_{p} \cdot\left(T_{z, i}-T_{z}\right)=\text { heat transfer due to the inter-zone air mixing. } \\
m_{\mathrm{inf}} \cdot c_{p} \cdot\left(T_{e x t}-T_{z}\right)=\text { heat transfer due to the outdoor air infiltration. }
\end{gathered}
$$

The term $\sum_{i=1}^{\text {Nsurface }} h \cdot A_{i} \cdot\left(T_{s i}-T_{z}\right)$ is affected by the short wave radiation from solar and internal sources, long wave radiation from/to other surfaces and endogenous sources and, mainly, by the heat conduction from/to the outside environment. In Figure 11, the various heat exchanges, on the outside and inside face of walls, are reported.

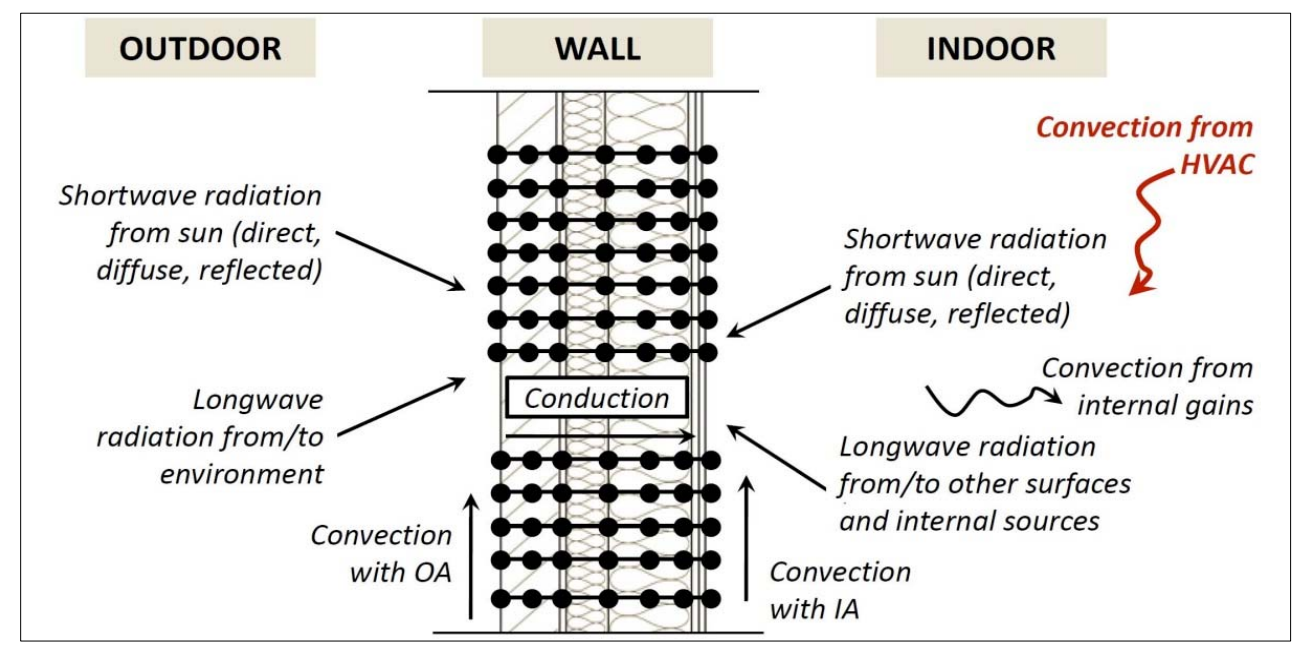

Figure 11: Heat balance on the outside and inside face of walls, according to the E+ engineering

The thermal bridges could be considered within the term $\sum_{i=1}^{N s j} \dot{Q}_{i}$ or, alternatively, into $\sum_{i=1}^{\text {Nsurface }} h \cdot A_{i} \cdot\left(T_{s i}-T_{z}\right)$.

The user will identify, in the third-party graphical interface, the position of each thermal bridge, defining each part of structure where the heat flow cannot be considered as one-dimensional. A new window will allow the definition of the discontinuity (i.e., materials, shape, boundary conditions). Then, the heat flow due to thermal bridges could be implemented - into the energy balances solved by EnergyPlus, such as summarized in (21), in two different ways:

a) the first possibility requires modification of the source code of EnergyPlus. When a thermal bridge is indicated by the user, the software will subtract its area by the common surface and create a new sub-surface. A code routine will create new structures analyzed such as described 
into this paper. Finally, the term $\sum_{i=1}^{\text {Nsurface }} h \cdot A_{i} \cdot\left(T_{s i}-T_{z}\right)$ will consider, among the walls, also the thermal bridges and their inner surface temperature.

b) The thermal bridge is solved, for each time-step, by using an autonomous sub-software, that works as described into this study. A simulation will be performed with reference to the whole season, using the hourly climatic data and a fixed set-point for the indoor air. In parallel, EnergyPlus will perform the traditional simulation. Then, the thermal bridge effect will be

considered as an additional load, by modifying the term $Q_{i}$ of (21), by defining a new "energy gain or loss" device, for example by using the module "other equipment". This equipment will provide a thermal flux equal to the sum of the zone thermal bridges, minus the heat flow already considered because the wall surfaces have been not modified by $\mathrm{E}+$. Then, a final $\mathrm{E}+$ simulation will performed.

In Figure 12, the two possibilities are schematized. The first will follow the steps 1, 23 and 4a. The second possibility will perform the steps $1,2,3$ and $4 \mathrm{~b}$. Surely, the second strategy will induce lower modification of the present structure of the engine. Anyway, it is applicable only if the indoor set-point conditions are fixed or scheduled. The solution doesn't work if the indoor temperatures free run.

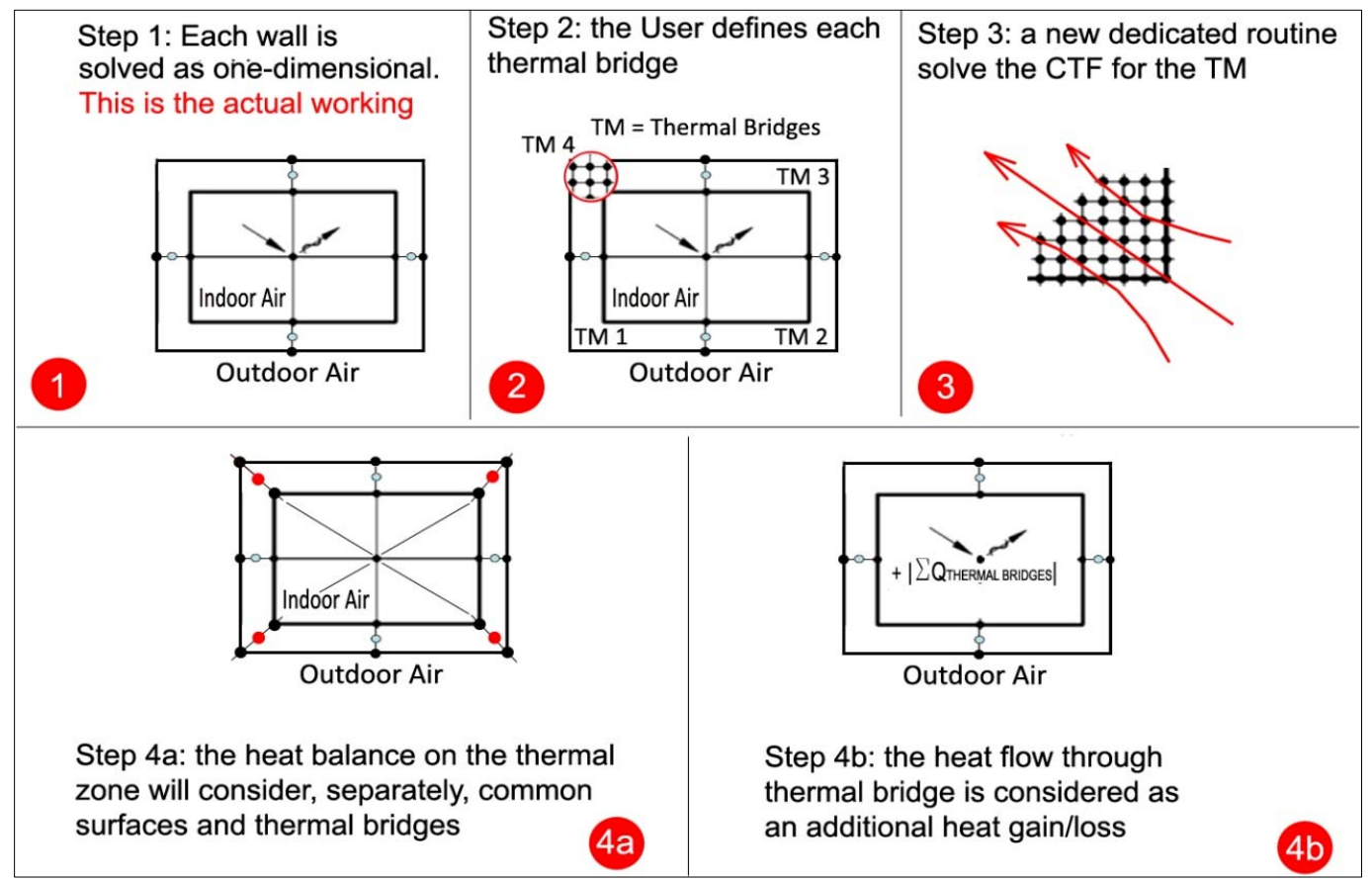

Figure 12: Possible implementation of the proposed method into dynamic energy simulation software

\section{CONCLUSIONS}

Several analyses have been carried out, in order to develop a numerical model that could be useful in the detection of heat flows through common thermal bridges. The studies were conducted at various levels in order to verify the stability of the solution as well as the required computational times, with the aim to offer a model useful for large audits, extended to the whole year. This target cannot be obtained with complex CFD codes, because of the high computational times required by these.

The studies were applied to a one-dimensional vertical wall and to a L-structured thermal bridge.

Using the developed model, it was found that the computation times are extremely low and the achievable results are, at the same time, quite satisfactory. In particular, the solution stability is commonly very high, regardless the chosen time-step. 
The final part of the paper proposes some suggestions for the integration of the proposed methods in numerical codes, like, for example, EnergyPlus, for the building energy diagnosis. The final target is the better simulation of the thermal behaviours and, therefore, the thermal energy needs of buildings.

Globally, the outcomes are very satisfactory, suggesting further deepening, presently carried out by the authors.

\section{NOMENCLATURE}

\begin{tabular}{|c|c|c|}
\hline Q & Heat Flow & {$[\mathrm{W}]$} \\
\hline CTF & Conduction Transfer Function & ---- \\
\hline A & Matrix $(n \times n)$ & ---- \\
\hline B & Matrix $(n x p)$ & ---- \\
\hline $\mathrm{C}$ & Matrix $(m \times n)$ & ---- \\
\hline $\mathrm{D}$ & Matrix $(m \times p)$ & ---- \\
\hline $\mathrm{c}_{\mathrm{v}}$ & Specific heat (constant volume) & {$[\mathrm{J} / \mathrm{kgK}]$} \\
\hline$c_{p}$ & Specific heat (constant pression) & {$[\mathrm{J} / \mathrm{kgK}]$} \\
\hline$e_{j}$ & Transfer function coefficient & ---- \\
\hline $\mathrm{e}^{\mathrm{A} \delta}$ & Exponential matrix & ---- \\
\hline $\mathrm{h}$ & Convective heat transfer coefficient & {$\left[\mathrm{W} / \mathrm{m}^{2} \mathrm{~K}\right]$} \\
\hline $\mathrm{k}$ & Thermal Conductivity & {$[\mathrm{W} / \mathrm{mK}]$} \\
\hline I & Matrix of Identity $(n \times n)$ & ---- \\
\hline $\mathrm{m}$ & Number of Outputs; & ---- \\
\hline $\mathrm{n}$ & Number of the state-variables; & ---- \\
\hline $\mathrm{p}$ & Number of Inputs; & ---- \\
\hline $\mathrm{R}_{\mathrm{j}}$ & Matrix of Coefficients $(n \times n)$; & ---- \\
\hline $\mathrm{S}_{\mathrm{j}}$ & Matrix $(m \times p)$ of the Transfer function coefficients & ---- \\
\hline $\mathrm{T}$ & Temperature & {$[\mathrm{K}]$} \\
\hline $\mathrm{T}_{\text {ext }}$ & Temperature (generic) & {$[\mathrm{K}]$} \\
\hline $\mathrm{T}_{\mathrm{si}}$ & Temperature of the inner surface & {$[\mathrm{K}]$} \\
\hline $\mathrm{T}_{\mathrm{z}}$ & Indoor air temperature of the considered thermal zone & {$[\mathrm{K}]$} \\
\hline $\mathrm{T}_{\mathrm{z}, \mathrm{i}}$ & Indoor air temperature of the generic thermal zone " $\mathrm{i}$ " & {$[\mathrm{K}]$} \\
\hline $\mathrm{u}$ & Vector of Inputs & ---- \\
\hline $\mathrm{x}$ & Vector of the state-variables & ---- \\
\hline $\mathrm{x}_{(\mathrm{i}, \mathrm{j})}$ & Element in row $\mathrm{i}$ and column $\mathrm{j}$ of the $\mathrm{X}$ matrix & ---- \\
\hline y & Vector of Outputs & ---- \\
\hline \multicolumn{3}{|c|}{ Greek letters } \\
\hline$\alpha$ & Name of CFD mesh (example 1: 6000 cells, example 2: 1500 cells) & {$\left[\mathrm{m}^{2} / \mathrm{s}\right]$} \\
\hline$\beta$ & Name of CFD mesh (example 1: 15000 cells, example 2: 4000 cells) & ---- \\
\hline$\zeta$ & Name of CFD mesh (example 1: 24000 cells, example 2: 6000 cells) & ---- \\
\hline$\gamma$ & Name of CFD mesh (example 2: 24000 cells) & ---- \\
\hline$\chi$ & Time $[\mathrm{s}]$ & ---- \\
\hline$\tau$ & Matrix $(n x p)$ & ---- \\
\hline$\Gamma_{1}$ & Matrix $(n x p)$ & ---- \\
\hline$\Gamma_{2}$ & Time step interval $[\mathrm{h}]$ & ---- \\
\hline$\delta$ & Density $\left[\mathrm{kg} / \mathrm{m}^{3}\right]$ & ---- \\
\hline$\rho$ & Exponential Matrix $(n \times n)=e^{\mathrm{A} \delta}$ & ---- \\
\hline$\Phi$ & Name of CFD mesh (example 1: 6000 cells, example 2: 1500 cells) & ---- \\
\hline
\end{tabular}

\section{REFERENCES}

Ali Alajmi. 2012. "Energy audit of an educational building in a hot summer climate." Energy and Buildings 47:122-130. 
Assem, E.O., A.A. Al-Mumin. 2010. "Code Compliance of Fully Glazed Tall Office Buildings in Hot Climate." Energy and Buildings 42(7):1100-1105.

Ascione, F., F. De Rossi, G.P. Vanoli. 2011. "Energy retrofit of historical buildings: Theoretical and experimental investigations for the modelling of reliable performance scenarios." Energy and Buildings 43(8):1925-1936.

Ascione F., N. Bianco, F. de Rossi, G. Turni, G.P. Vanoli. 2012. "Different methods for the modelling of thermal bridges into energy simulation programs: Comparisons of accuracy for flat heterogeneous roofs in Italian climates." Applied Energy 97:405-418.

ASHRAE, ASHRAE 2005. Handbook of Fundamentals, American Society of Heating Refrigerating and Air Conditioning Engineers, Atlanta, GA.

Ben Larbi, A. 2005. "Statistical modeling of heat transfer for thermal bridges of buildings." Energy and Buildings 37:945-951.

Ben-Nakh, A.E. 2002. "Minimizing thermal bridging through window systems in buildings of hot regions." Applied Thermal Engineering 22:989-998.

Brogan, W.L. 1990. Modern control theory. 3rd Edition. Prentice Hall, New Jersey.

Ceylan, H.T. and G.E. Meyers 1980. "Long-time solutions to heat conduction transient with time-dependent inputs." ASME Journal of Heat Transfer 102:115-120.

CSTB. 2000. Centre Scientifique et Technique du Bâtiment. Réglementation thermique, Chapter 2, Paris.

Déque, F., F. Ollivier, A. Poblador. 2000. "Grey boxes used to represent buildings with a minimum number of geometric and thermal parameters." Energy and buildings 31:29-35.

Déque, F., F. Ollivier, J.J. Roux. 2001. "Effect of 2D modeling thermal bridges on the energy performance of buildings Numerical application on the Matisse apartment." Energy and buildings 33:583-587.

Duffie, J.A., W.A. 2006. Solar Engineering of thermal process, 3rd Edition, John Wiley \& Sons, Hoboken, New Jersey.

ANSYS. 2005. Fluent 6.2. User manual, ANSYS Inc.

Gao, Y., J.J. Roux, H.H. Zhao, J. Jiang. 2008. "Dynamical building simulation: A low order model for thermal bridges losses." Energy and Buildings 40:2236-2243.

Hirsch, J.J. \& Associates. 2005. Software DOE, release 2.2.2005. Lawrence Berkeley National Laboratory Simulation Research Group, Berkeley CA, USA.

Hittle, D.C. 1981. "Calculating building heating and cooling loads using the frequency response of multilayered slabs." PhD Thesis, University of Illinois at Urbana-Champaign, IL.

Hittle, D.C., R. Bishop. 1983. "An improved root-finding procedure for use in calculating transient heat flow through multilayer slabs." International Journal of Heat Mass Transfer 26 (1):1685-1693.

Klein, S.A. et. al. 2000. TRNSYS - TRaNsient SYstem Simulation Program - Solar Energy Laboratory, University of Wisconsin, Madison, USA.

Kosny, J. and J.E. Christian. 1995. "Thermal evaluation of several configurations of insulation and structural materials for some metal studs." Energy and Buildings 22:157-163.

Kosny, J. and E. Kossecka, "Multi-dimensional heat transfer through complex buildings envelope assemblies in hourly energy simulation programs." Energy and Buildings 34:445-454.

Marshall, S.A. 1966. "An approximate method for reducing the order of a linear system." Control 10:642643.

Martin K., A. Erkoreka, I. Flores, M. Odriozola, J.M. Sala. 2011. "Problems in the calculation of thermal bridges in dynamic conditions". Energy and Buildings 43:529-35.

MathWorks. 2006. Matlab - MATrix LABoratory - MATLAB Version 7.3, User's Guide 2006.

Moore, B. 1981. "Principal Component Analysis in Linear Systems: Controllability, Observability and Model Reduction", IEEE Transactions on Automatic Control AC-26 (1):17-32.

Moralesa, M.P., M.C. Juáreza, L.M. López-Ochoaa and J. Doménecha. 2011. "Study of the geometry of a voided clay brick using rectangular perforations to optimize its thermal properties." Applied Thermal Engineering 31:2063-2065.

Ouyang, K. and F. Haghighat. 1991. "A procedure for calculating thermal response factors of multi-layered walls-state space method." Building and Environment 26:173-177.

Pisello, A.L., J.E. Taylor, X. Xu, F. Cotana. 2012. "Inter-building effect: Simulating the impact of a network of buildings on the accuracy of building energy performance predictions." Building and Environment $58: 37-45$.

Renon, O. 2002. "Thermal bridges modeling in EnergyPlus." Building energy simulation user news, 23 (4) (2002). 
Seem, J.E., Modelling of heat transfer in buildings, Ph.D. Thesis, University of Wisconsin, 1987

Seem, J.E., S.A. Klein, W.A. Beckman J.W. Mitchell. 1989. "Transfer function for efficient calculation of multidimensional transient heat transfer." ASME Journal of Heat Transfer 111:5-12.

Stephenson, D.G., G.P. Mitalas. 1971. Calculation of heat conduction transfer functions for multi-layered slabs." ASHRAE Transactions 77, part II.

U.S. Department of Energy. 2010. Building Technology Program, EnergyPlus. Energy Efficiency and Renewable Energy Office. Available from:http://apps1.eere.energy.gov/buildings/energyplus/.

White, F.M. 1988. Heat and mass transfer, Addison Wesley, Reading, MA.

Zheng, B., G. Wang. 2005. "Leverrier's algorithm and Cayley-Hamilton theorem for 2-D system", Applied Mathematics and Computation, (160):725-738.

\section{AUTHOR BIOGRAPHIES}

FABRIZIO ASCIONE studied Architecture at the University of Naples, Italy. From 2006 to 2009 he has been a Ph.D. student at the graduate school of Engineering of Mechanical Systems. In 2009, he received his Ph.D. for his work about Passive and Low-Energy Heating and Cooling strategies. Since March 2010, he is Grant Researcher at the University of Sannio in Benevento, with a scientific assignment called "Theoretical tools for the evaluation of thermal behavior, under single- and two-dimensional variable regimes, of innovative housing envelope solutions for the reduction of the primary energy requests". He is author/coauthor of various papers in regard to energy efficiency in buildings. His email address is fabrizio.ascione@unina.it.

NICOLA BIANCO is Associate Professor of Applied Thermodynamics at the Facolty of Engineering of the University of Naples Federico II. He got a degree in Mechanical Engineering, cum laude, obtaining the Ph.D. degree in Thermo-mechanical Engineering. He has been in charge of research projects funded by the National Agency for Energy and Environment (ENEA), Italian Ministry for Instruction and Research, Campania Region. He has been the author or the co-author of numerous scientific papers published in national and international journals and conference proceedings. His research fields, by means of analytical, numerical and experimental techniques, are natural and mixed convection in channels and cavities, heat conduction in solids with moving heat source, thermo-energy design of solar systems, analysis of the building envelope. His email address is nicola.bianco@unina.it.

FILIPPO DE ROSSI is Full Professor, for the Italian Scientific Sector Applied Thermodynamics and Heat Transfer for Buildings, at the Engineering Faculty of the Sannio University in Benevento (Italy). Since 2006, he is also the Dean of the Engineering Faculty. He has been the chief of various low-impact research projects for transferring technological investigation to both public and private companies. He is Vice-President of the B2 Commission (Refrigerating Plant), of the International Institute of Refrigeration. In regard to the scientific research, F. de Rossi carried out studies in the fields of solar energy, thermodynamic analysis of systems and components for refrigeration, air-conditioning systems for buildings and energy saving strategies for the building sector, both with reference to the building envelope and the HVAC systems. He is author and/or co-author of many scientific papers, on national and international journals, presented at national and international conferences, books or chapter of handbooks. His email address is derossi@unisannio.it.

GIUSEPPE PETER VANOLI is Associate Professor, for the Italian Scientific Sector Applied Thermodynamics and Heat Transfer for Buildings, at the Engineering Faculty of the Sannio University in Benevento (Italy). In January 2002, he achieved the the Ph.D. in Energetics. In March 2002, he awarded a researcher position for Applied Thermodynamics and Heat Transfer, at University of Sannio. In regard to the research activities, he has been author or co-author of numerous papers, published in national and international journals and conference proceedings. These studies are carried out with analytic, numerical and experimental approaches, with reference to the following topics: a) theoretical and experimental analysis, for assessing the energy performance of the system building-HVAC devices, b) refrigeration (heat exchange coefficient and the local pressure loss, during evaporation of new refrigerants, vertical freezers, heat exchangers of micro-channels). His email address is vanoli@unisannio.it. 